\title{
DEMO ABSTRACT - Applications of the CPAL Language to Model, Simulate and Program Cyber-Physical Systems
}

\author{
Loïc Fejoz \\ RealTime-at-Work (RTaW), France \\ loic.fejoz@realtimeatwork.com
}

\author{
Nicolas Navet, Sakthivel Manikandan Sundharam \\ and Sebastian Altmeyer \\ University of Luxembourg, Luxembourg \\ firstname.lastname@uni.lu
}

CPAL is a new language to model, simulate, verify and program Cyber-Physical Systems (CPS). CPAL serves to describe both the functional behaviour of activities (i.e., the code of the function itself) as well as the functional architecture of the system (i.e., the set of functions, how they are activated, and the data flows among the functions). CPAL is meant to support two use-cases. Firstly, CPAL is a development and design-space exploration environment for CPS with main features being the formal description, the editing, graphical representation and simulation of CPS models. Secondly, CPAL is a real-time execution platform. The vision behind CPAL is that a model is executed and verified in simulation mode on a workstation and the same model can be later run on an embedded board with a timing-equivalent run-time behaviour. The design and development of CPAL have been organized around a set of realistic case-studies that will be demonstrated during the demonstration session. The CPAL case studies and experiments are inspired from the research and teaching carried out at University of Luxembourg, and RTAW's projects with partner and customer companies. 